\title{
Effect of Toll-like receptor 4 deficiency on clinical severity and expression of Th1/Th2/Th17-associated cytokines in a murine model of experimental autoimmune neuritis
}

\author{
Li-Juan Wang ${ }^{1,2,3,4}$, Jie Zhu ${ }^{5,6}$, Xiu-Juan Wu ${ }^{6}$, Ting Li ${ }^{6}$, Chun-Jiao Yang ${ }^{1,2,3,4}$, Xi-Xiong Kang ${ }^{1,2,3,4}$, \\ Hong-Liang Zhang, ${ }^{6,7}$, Guo-Jun Zhang ${ }^{1,2,3,4}$
}

\author{
${ }^{1}$ Laboratory Diagnosis Center, Beijing Tiantan Hospital, Capital Medical University, \\ Beijing, China \\ ${ }^{2}$ China National Clinical Research Center for Neurological Diseases, Beijing Tiantan \\ Hospital, Capital Medical University, Beijing, China \\ ${ }^{3}$ Monogenic Disease Research Center for Neurological Disorder, Beijing, China \\ ${ }^{4}$ Precision Medicine Research Center for Neurological Disorder, Beijing, China \\ ${ }^{5}$ Department of Neurobiology, Care Sciences and Society, Karolinska Institute, \\ Stockholm, Sweden \\ ${ }^{6}$ Neuroscience Center, Department of Neurology, the First Hospital of Jilin University, \\ Changchun, China \\ ${ }^{7}$ Department of Life Sciences, the National Natural Science Foundation of China, \\ Beijing, China
}

Submitted: 24 October 2018

Accepted: 25 November 2018

Arch Med Sci

DOI: https://doi.org/10.5114/aoms.2020.94982

Copyright $\odot 2020$ Termedia \& Banach

\begin{abstract}
Introduction: The aim was to observe the effect of Toll-like receptor 4 (TLR4) deficiency on clinical severity and expression of Th1/Th2/Th17-associated cytokines in experimental autoimmune neuritis (EAN).

Material and methods: We selected C57BL/10 wild type (WT) mice and TLR4 knockout (KO) mice with the C57BL/10 background for induction of the EAN model by immunizing mice twice (days 0 and 8) via subcutaneous injection of $180 \mu \mathrm{g}$ PO peptide 180-199 emulsion in $25 \mu \mathrm{l}$ of PBS and $0.5 \mathrm{mg}$ Mycobacterium tuberculosis (Difco, USA) in $25 \mu \mathrm{l}$ of Freund's incomplete adjuvant into the back of mice. The concentrations of serum cytokines (IL-2, IL-4, IL-6, IL-10, IL-17A, IFN- $\gamma$ and TNF) were determined using the Ms Th1/Th2/Th17 CBA kit. Results: We found that TLR4 deficiency could attenuate the clinical severity and delay the onset of EAN. Moreover, our data showed that the sera levels of IFN- $\gamma$, TNF, IL- 6 and IL-17A were elevated in the WT mice with EAN when compared with the naive WT mice, but only the production of IL-17A was significantly lower in the TLR4 KO mice with EAN than in their WT counterparts. Conclusions: Based on these findings, TLR4 may contribute to the pathogenesis of EAN by regulating Th17 cells and the production of Th17-associated factors. However, the exact mechanism remains unclear and more evidence is needed to elucidate its role in EAN.
\end{abstract}

Key words: cytokine, Guillain-Barré syndrome, Toll-like receptor 4, experimental autoimmune neuritis.

\section{Introduction}

Guillain-Barré syndrome (GBS) is a rare autoimmune disorder which attacks the peripheral nervous system, characterized by rapidly progres-

\author{
Corresponding author: \\ Dr. Guo-Jun Zhang \\ Laboratory Diagnosis Center \\ Beijing Tiantan Hospital \\ Capital Medical University \\ Beijing, China \\ Phone: 01059978452 \\ E-mail:571233145@qq.com
}


sive symmetrical paralysis in the limbs, sensory dysfunction, and areflexia [1-3]. Experimental autoimmune neuritis (EAN) is a counterpart animal model for studying GBS. To date, the pathogenesis of GBS/EAN has been extensively studied, including cellular and humoral immune responses [4, 5], molecular mimicry, the Th1/Th2/Th17 paradigm [6-9] and Toll-like receptors (TLRs) [10, 11], etc., but is not completely clear.

Toll-like receptors are predominantly expressed on the immune cells, which can induce a wide variety of immune responses to specific pathogens $[12,13]$. Increasing evidence suggests that TLRs are involved in the pathogenesis of GBS from different aspects including gene, patients with GBS as well as EAN research. Nyati et al. reported that inflammatory genetic polymorphisms may predict risk and susceptibility for development of GBS [14], and they also found that TLR4 (Asp299Gly) polymorphism is related to increased susceptibility to GBS [15]. Du [16] and Wang et al. [10] revealed up-regulated expression of TLR2, 4 and 9 and their relational signaling molecules in patients with GBS. A significant increase of TLR2, TLR6 and TLR1 1 was observed in the C57Bl/6J EAN mice by Gries [11]. Meanwhile, Deng et al. [17] found TLR4 and TLR9 mRNA expressed highly in Lewis rats with EAN.

Moreover, it is generally accepted that interleukin (IL)-17 plays pivotal roles in autoimmune diseases which facilitates the generation of many chemokines, cytokines, and growth factors. However, whether there is an association of Th17 cells or IL-17 cytokines with TLR4 in EAN remains poorly documented. Wang et al. [18] found that IL-17A participated in development of spontaneous pulmonary emphysema which was caused by Toll-like receptor 4 mutation.

Therefore, to observe the effect of Toll-like receptor 4 (TLR4) deficiency on clinical severity and expression of Th1/Th2/Th17-associated cytokines, we selected C57BL/10 mice and TLR4 knockout (KO) mice with the C57BL/10 background for induction of the EAN model. The results are reported as follows.

\section{Material and methods}

\section{Animals}

C57BL/10 mice and TLR4 KO mice [19] with the C57BL/10 background were purchased from the Model Animal Research Center of Nanjing University (Nanjing, China). Male mice, 4-5 weeks old (18-20 g), were used for the study. All mice were housed in a $12 / 12$ light-dark environment at $25^{\circ} \mathrm{C}$ in the Animal Care Facility of Jilin University according to institutional guidelines.

\section{Antigen}

The P0 peptide 180-199 (SSKRGRQTPVLYAMLDHSRS) of murine peripheral myelin protein PO was produced according to the following steps: it was first synthesized by the 9-fluorenylmethoxycarbonyl (Fmoc) solid-phase procedure; then purified by HPLC using a Vydac reverse-phase column (Grace Vydac, Hesperia, CA, USA); and finally analyzed by MALDI-time of flight (TOF) mass spectrometry (Cambridge Research Biochemicals, Billingham, UK).

\section{Induction of experimental autoimmune} neuritis and evaluation of clinical signs

We induced EAN by immunizing mice via subcutaneous injection of $180 \mu \mathrm{g}$ of PO peptide 180-199 (SSKRGRQTPVLYAMLDHSRS) [20] (GenScript, USA) emulsion in $25 \mu \mathrm{l}$ of PBS and $0.5 \mathrm{mg}$ of Mycobacterium tuberculosis (Difco, USA) in $25 \mu \mathrm{l}$ of Freund's incomplete adjuvant (Sigma Aldrich, USA). On days -1 and +1 after immunization, 400 and 300 ng of pertussis toxin (Merck Millipore, Germany), respectively, was administered intravenously (via tail veins). A blinded protocol was used to assess clinical signs of mice with EAN according to the following criteria: normal (score $=0)$, reduced tone of the tail $($ score $=1)$, flaccid tail (score $=2)$, abnormal gait $($ score $=3$ ), ataxia (score $=4)$, mild paraparesis (score $=5$ ), moderate paraparesis (score $=6$ ), severe paraparesis (score $=7$ ), intermediate clinical signs (score $=0.5$ ). To observe the roles of TLR4 and Th1/Th2/Th17-associated cytokines in the EAN, the animals were grouped as follows: Naive WT group, EAN WT group, EAN TLR4 KO group, which were sacrificed at the peak of clinical EAN, i.e., at day 16 post-immunization. The sera were quickly isolated and stored at $-80^{\circ} \mathrm{C}$.

\section{Serum cytokine measurement by cytometric bead array}

Concentrations of serum cytokines (IL-2, IL-4, IL6, IL-10, IL-17A, interferon [IFN]- $\gamma$ and tumor necrosis factor [TNF]) were determined using the Ms Th1/ Th2/Th17 CBA kit (BD Biosciences, USA) according to the manufacturer's instructions. We analyzed beads by flow cytometry using an FACSCalibur flow cytometer and CellQuest software (BD Biosciences, USA). We analyzed data with a spreadsheet developed by BD Biosciences that used a four-parameter logistic curve-fitting model [21].

In our study, all experiments were repeated three times, and the averages were used for statistical analysis. Moreover, our study has been approved by the Ethics Committee of Beijing Tiantan Hospital, Capital Medical University, and the ethical review number is KY2016-052-02. 


\section{Statistical analysis}

Statistical analysis was performed using SPSS 20.0 for Windows (SPSS Inc., Chicago, IL, USA). All results obtained are presented as mean values \pm standard deviation (SD). Differences of the Th1/ Th2/Th17 cytokines between the WT mice, the WT mice with EAN and the TLR4 KO mice with EAN were compared using the one-way analysis of variance (ANOVA) or the Mann-Whitney $U$ test. To test for differences of clinical scores between TLR4 KO mice and their WT counterparts, two independent-samples $t$-test or the Mann-Whitney $U$ test was applied. $P<0.05$ was considered statistically significant.

\section{Results}

Toll-like receptor 4 deficiency alleviates the clinical severity of experimental autoimmune neuritis

As shown in Figure 1, the onset of EAN in WT mice ranged between days 7 and 8 p.i. In contrast, the TLR4 KO mice experienced a later onset of EAN, i.e., at days 9-10 p.i. From day 9 p.i., the clinical scores of TLR4 KO mice were evidently milder than those of their WT counterparts with the exception of day 13 post-immunization. Differences between them were compared using two independent-samples $t$-test or the Mann-Whitney $U$ test. ${ }^{* *} P<0.01,{ }^{* * *} p<0.001$.

\section{Toll-like receptor 4 deficiency decreases the production of IL-17A}

To prove the involvement of serum cytokines in the pathogenesis of EAN, mice were sacrificed at the peak period of disease (day 16 p.i.) and the sera were collected rapidly for Th1/Th2/ Th17 cytokine (including IL-4, IL-6, IL-10, IFN- $\gamma$, TNF, IL-17A) detection by cytometric bead array (CBA). Compared to the WT mice, the WT mice with EAN showed significant elevation in IL-6, IL-17A, IFN- $\gamma$ and TNF. However, there were no significant differences in IL-4 and IL-10 concentrations between them. Furthermore, the results suggest that only sera IL-17A was statistically significantly higher in WT mice with EAN than that of their TLR4 KO counterparts $(p<0.05)$, but there was no difference in IFN- $\gamma$, TNF, IL-4, IL-6, IL-10 concentrations between them (Figure 2). Differences between them were compared using the one-way analysis of variance (ANOVA) or the Mann-Whitney $U$ test. ${ }^{*} P<0.05,{ }^{* *} p<0.01$, NS - no significance.

\section{Discussion}

At present, the GBS animal model of EAN, which can mimic the human GBS, is used widely. In our study, we induced EAN in C57BL/10 and TLR4 KO

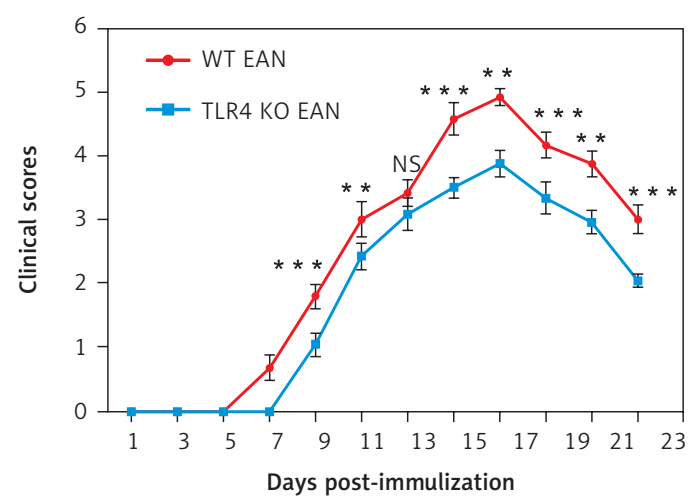

Figure 1. Clinical scores of Toll-like receptor 4 (TLR4) knockout (KO) experimental autoimmune neuritis (EAN), wild type (WT) EAN mice and naïve WT mice. Experimental autoimmune neuritis was induced by immunization with P0 peptide 180-199 in combination with pertussis toxin (PTX) and Freund's complete adjuvant (FCA). All mice received 400 and $300 \mathrm{ng}$ PTX intravenously on days -1 and +1 p.i., respectively. Using a blinded protocol, two different examiners evaluated clinical severity of EAN immediately after immunization (day 0 p.i.) and thereafter every day until day 24. After 24 days p.i., the clinical course began to recover. Results from the three groups are presented as mean values \pm SD ( $n=6$ in every group). From day 9 p.i., the clinical signs of TLR4 KO mice were significantly milder than those of their WT counterparts with the exception of day 13 postimmunization. Differences between them were compared using two independent-samples t-test or the Mann-Whitney $U$ test. ${ }^{* *} P<0.01,{ }^{* * *} p<0.001$, NS - no significance

mice. We found that the onset of EAN in TLR4 KO mice was significantly delayed. Meanwhile, we noted a trend of milder clinical signs of EAN in TLR4 KO mice as well. Further, we found that TLR4 deficiency only reduced the production of Th17 cytokine IL-17A, but did not downregulate the expression of other Th1/Th2/ Th17-associated cytokines, such as IL-4, IL-6, IL-10, IFN- $\gamma$, and TNF. Consistently with our study, Zhang et al. [22] found that IL-17(+) cells might contribute to the pathogenesis of EAN by examining the spatiotemporal expression of IL-17 using immunohistochemistry and RT-PCR, and analyzed the IL-17(+) cell proportion in blood and lymph nodes using flow cytometry. Combined with our data, we suppose that TLR4 deficiency may mitigate experimental autoimmune neuritis via modification of the Th17/IL17 responses. The exact conclusion needs to be explored further, such as detecting the spatiotemporal expression of IL-17 using immunohistochemistry or RT-PCR in TLR-4 mice.

Additionally, the study revealed that reduced expression of IL-6, IL-12p40, and IL-17A in nuclear translocation of RelA EAN knockout mice and obviously elevated Tregs were found in TLR4 KO mice as compared to wild-type mice in the recovery phase. A study found that cytokines such as IL-6, IL-17A, Th/Tregs and TLRs may be involved 
A

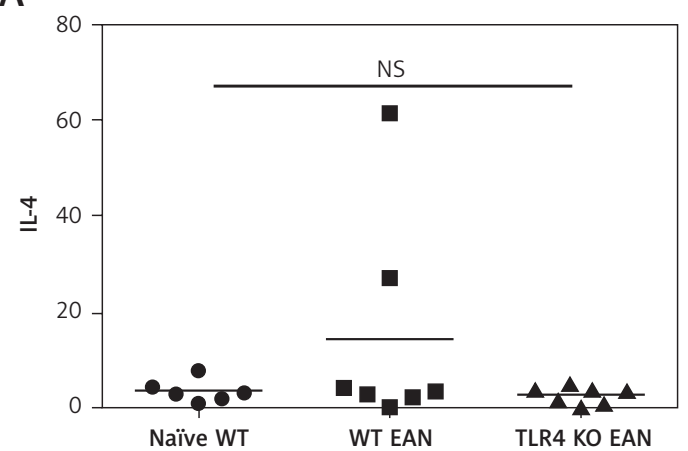

C

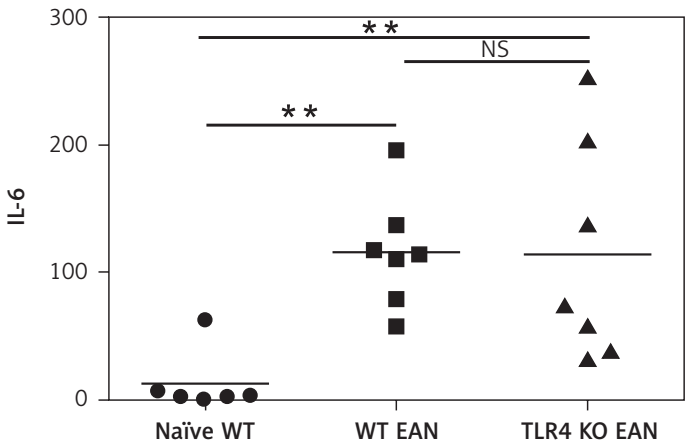

$\mathrm{E}$

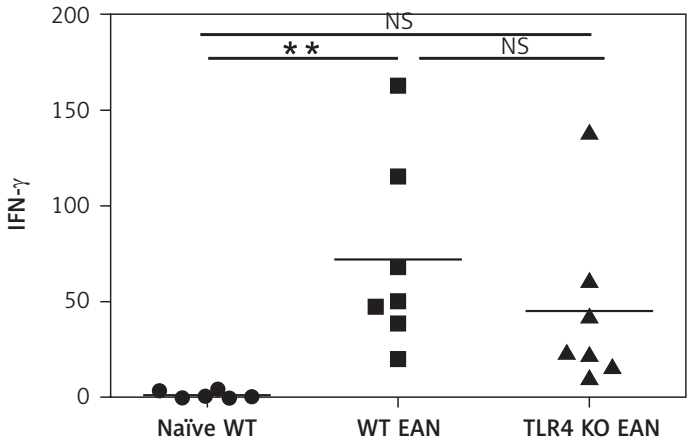

B

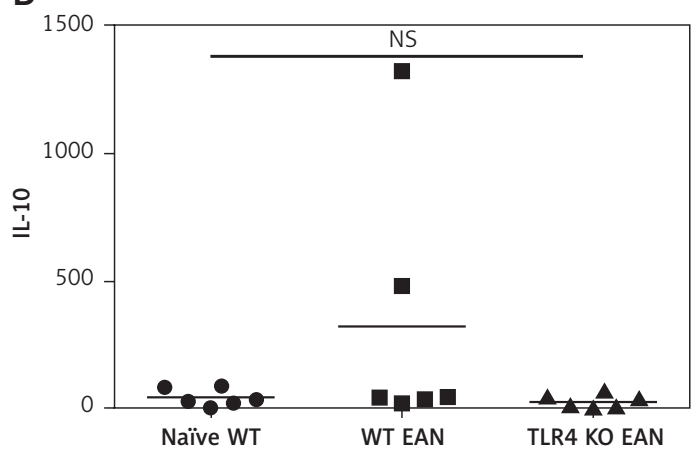

D

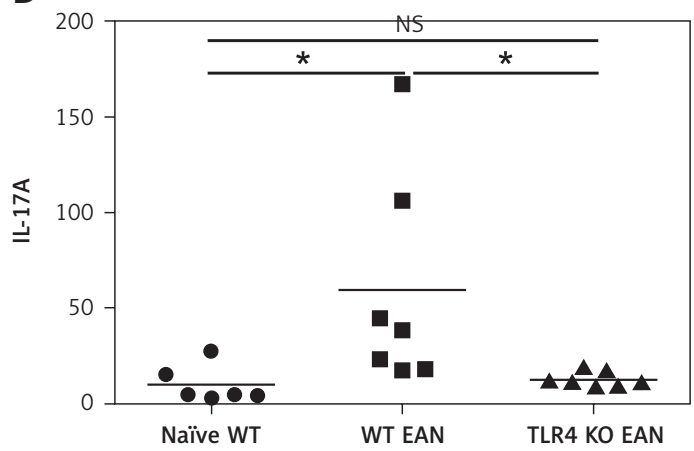

$\mathbf{F}$

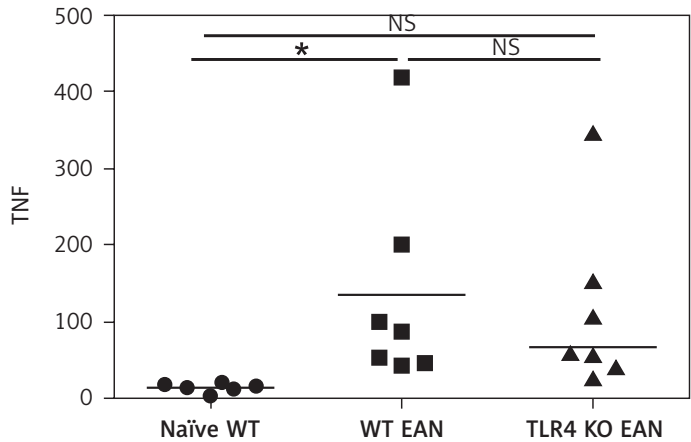

Figure 2. Expression of interleukin (IL)-4, IL-6, IL-10, IL-17A, interferon (IFN)- $\gamma$ and tumor necrosis factor (TNF) of sera from naïve wild type (WT), WT experimental autoimmune neuritis (EAN) and Toll-like receptor 4 (TLR4) knockout (KO) EAN mice. Mice were sacrificed at the peak period of disease (day 16 p.i.) and the sera were collected rapidly for Th1/Th2/Th17 cytokine detection by cytometric bead array (CBA). Data are represented as means. Except for IL-4 and IL-10, the expression of IL-6, IL-17A, IFN- $\gamma$ and TNF was significantly higher in WT EAN mice compared with naïve WT mice. However, only the expression of IL-17A was significantly higher in the WT EAN mice compared with the TLR4 KO EAN mice. Differences between them were compared using two independent-samples $t$-test, the Mann-Whitney $U$ test or one-way analysis of variance (ANOVA). ${ }^{* *} P<0.05$, ${ }^{* *} p<0.01$, NS - no significance

in the disease progression [23]. However, our research indicated that there may be a link between TLR4 and IL17/Th17 in the pathogenesis of GBS.

Generally, researchers suggest that a disturbance of the cytokines Th1/Th2/Th17/Treg balance is one of the main pathological mechanisms in GBS or EAN $[9,24]$. Li and colleagues proved that the amount of Th1/Th2/Th17 cells was obviously increased in GBS [24]. Given the important roles of cytokines in the immunopathogenesis in both
GBS and EAN, we sought to examine a broad spectrum of serum cytokines in WT and TLR4 KO mice. In line with investigations by Matsui et al. [25], we observed that the serum expression of Th1 cytokines IFN- $\gamma$, TNF, IL- 6 and Th17 cytokine IL-17A were increased in WT EAN mice while expression of Th2 cytokines IL-4 and IL-10 was not significantly different when compared with WT mice. Similar results were also obtained in other studies $[26,27]$ However, Zhang et al. observed aggravated clinical scores despite an alleviated systemic Th1 immune 
response in IFN- $\gamma$ deficient mice with EAN [28]. Interestingly, our current data showed that serum IL-17A and clinical score were lower in TLR4 KO mice with EAN than in their WT counterparts, which indicated a certain link between the TLR4 signaling pathway and Th1/Th2/Th17 responses in C57BL/10 mice with EAN. Some studies demonstrated that the TLR4 mRNA is highly expressed on Th17 cells and the TLR4 signaling pathway increases Th17 activation and persistence but weakens Th1 responses [29, 30]. Moreover, Xu and colleagues [31] reported that TLR4 signaling inhibited Th1 cell differentiation by suppressing the STAT1 pathway and facilitated Th17 cell differentiation by activating the STAT3 pathway in the mouse model of MPE. Increasing evidence shows that TLR4 activation plays a critical role in the development of Th17cells and the expression of Th17-associated cytokines under normal or infectious situations [29, 32, 33]. Bhan and his team showed that Gam-negative bacterium induced the expression of IL-17 by activating TLR4 [34]. The attenuation of IL- $1 \beta$, TNF- $\alpha$, IFN- $\gamma$, IL-17, and IL-23 was found in the lungs of TLR4-mutant mice after vaccination with pertussis vaccines [35].

In summary, TLR4 deficiency could attenuate the clinical severity and delay the onset of EAN. Meanwhile, it also reduced the production of the inflammatory cytokine IL-17A. Based on these findings, TLR4 may contribute to the pathogenesis of EAN by regulating Th17 cells and the level of Th17-associated factors. However, the exact mechanism remains unclear and more evidence is needed to elucidate its role in EAN.

\section{Acknowledgements}

The authors would like to thank Chao Zheng, Caiyun Liu and Qingqing Cao, who have supported the author, with sincere gratitude.

This study was supported by a Contract grant sponsor, Beijing Natural Science Foundation, 7142051 (Zhang GJ); the application research of the Capital Clinical Characteristics, Z151100004015144 (Zhang GJ).

\section{Conflicts of interest}

The authors declare no conflict of interest.

\section{References}

1. Goodfellow JA, Willison HJ. Guillain-Barré syndrome: a century of progress. Nat Rev Neurol 2016; 12: 723-31.

2. Yuki N, Hartung HP. Guillain-Barré syndrome. N Engl J Med 2012; 366: 2294-304.

3. Doets AY, Jacobs BC, van Doorn PA. Advances in management of Guillain-Barré syndrome. Curr Opin Neurol 2018; 31: 541-550.

4. Bourque P, Chardon JW, Massie R. Autoimmune peripheral neuropathies. Clin Chim Acta 2015; 449: 37-42.
5. Li H, Li XL, Zhang M, et al. Berberine ameliorates experimental autoimmune neuritis by suppressing both cellular and humoral immunity. Scand I Immunol 2014; 79: 12-9.

6. Wu X, Wang J, Liu K, Zhu J, Zhang HL. Are Th17 cells and their cytokines a therapeutic target in Guillain-Barré syndrome? Expert Opin Ther Targets 2016; 20: 209-22.

7. Zhang HL, Wu L, Wu X, Zhu J. Can IFN-gamma be a therapeutic target in Guillain-Barré syndrome? Expert Opin Ther Targets 2014; 18: 355-63.

8. Wang X, Ma C, Wu J, Zhu J. Roles of T helper 17 cells and interleukin-17 in neuroautoimmune diseases with emphasis on multiple sclerosis and Guillain-Barré syndrome as well as their animal models. J Neurosci Res 2013; 91: 871-81.

9. Zhang HL, Zheng XY, Zhu J. Th1/Th2/Th17/Treg cytokines in Guillain-Barré syndrome and experimental autoimmune neuritis. Cytokine Growth Factor Rev 2013; 24: 443-53.

10. Wang YZ, Liang QH, Ramkalawan $\mathrm{H}$, et al. Expression of Toll-like receptors 2, 4 and 9 in patients with Guillain-Barré syndrome. Neuroimmunomodulation 2012; 19: 60-8.

11. Gries M, Davies L, Liu Y, et al. Response of Toll-like receptors in experimental Guillain-Barré syndrome: a kinetic analysis. Neurosci Lett 2012; 518: 154-60.

12. Hans M, Hans VM. Toll-like receptors and their dual role in periodontitis: a review. J Oral Sci 2011; 53: 263-71.

13. O'Neill LA. When signaling pathways collide: positive and negative regulation of toll-like receptor signal transduction. Immunity 2008; 29: 12-20.

14. Nyati KK, Prasad KN. Role of cytokines and Toll-like receptors in the immunopathogenesis of Guillain-Barré syndrome. Mediators Inflamm 2014; 2014: 758639.

15. Nyati KK, Prasad KN, Verma A, et al. Association of TLR4 Asp299Gly and Thr399lle polymorphisms with Guillain-Barré syndrome in Northern Indian population. J Neuroimmunol 2010; 218: 116-9.

16. Du Y, Zhang G, Zhang Z, et al. Toll-like receptor-2 and -4 are involved in the pathogenesis of the Guillain-Barre syndrome. Mol Med Rep 2015; 12: 3207-13.

17. Deng YN, Zhou WB. Expression of TLR4 and TLR9 mRNA in Lewis rats with experimental allergic neuritis. Neuroimmunomodulation 2007; 14: 337-43.

18. Wang QQ, Yang HZ, Liu HZ, et al. Interleukin-17A is involved in development of spontaneous pulmonary emphysema caused by Toll-like receptor 4 mutation. Acta Pharmacol Sin 2011; 32: 1045-54.

19. Petkov PM, Cassell MA, Sargent EE, et al. Development of a SNP genotyping panel for genetic monitoring of the laboratory mouse. Genomics 2004; 83: 902-11.

20. Gonsalvez DG, Fletcher JL, Yoo SW, et al. A simple approach to induce experimental autoimmune neuritis in C57BL/6 mice for functional and neuropathological assessments. J Vis Exp 2017. doi: 10.3791/56455.

21. Gabhann JN, Higgs R, Brennan K, et al. Absence of SHIP-1 results in constitutive phosphorylation of tank-binding kinase 1 and enhanced TLR3-dependent IFN-beta production. J Immunol 2010; 184: 2314-20.

22. Zhang ZY, Zhang Z, Schluesener HJ. FTY720 attenuates lesional interleukin-17(+) cell accumulation in rat experimental autoimmune neuritis. Neuropathol Appl Neurobiol 2009; 35: 487-95.

23. Brunn A, Mihelcic M, Carstov M, et al. Toll-like receptor 2, toll-like receptor 4 , myeloid differentiation response gene 88 , and toll-IL-1 receptor domain-containing adaptor-inducing interferon-gamma (TRIF) selectively regulate susceptibility of P0106-125-induced murine 
experimental autoimmune neuritis. Am J Pathol 2017; 187: 42-54.

24. Li S, Jin T, Zhang HL, et al. Circulating Th17, Th22, and Th1 cells are elevated in the Guillain-Barré syndrome and downregulated by IVIg treatments. Mediators Inflamm 2014; 2014: 740947.

25. Matsui H, Ohgomori T, Natori T, et al. Keratan sulfate expression in microglia is diminished in the spinal cord in experimental autoimmune neuritis. Cell Death Dis 2013; 4: e946.

26. Zhang J, Dong H, Li B, Li CY, Guo L. Association of tumor necrosis factor polymorphisms with Guillain-Barré syndrome. Eur Neurol 2007; 58: 21-5.

27. Liang SL, Wang WZ, Huang S, Wang XK, Zhang S, Wu Y. Th17 helper cell and T-cell immunoglobulin and mucin domain 3 involvement in Guillain-Barré syndrome. Immunopharmacol Immunotoxicol 2012; 34: 1039-46.

28. Zhang HL, Azimullah S, Zheng XY, et al. IFN-gamma deficiency exacerbates experimental autoimmune neuritis in mice despite a mitigated systemic Th1 immune response. J Neuroimmunol 2012; 246: 18-26.

29. Reynolds JM, Pappu BP, Peng J, et al. Toll-like receptor 2 signaling in CD4(+) T lymphocytes promotes $\mathrm{T}$ helper 17 responses and regulates the pathogenesis of autoimmune disease. Immunity 2010; 32: 692-702.

30. Reynolds JM, Martinez GJ, Chung Y, Dong C. Toll-like receptor 4 signaling in $T$ cells promotes autoimmune inflammation. Proc Natl Acad Sci U S A 2012; 109: 13064-9.

31. Xu QQ, Zhou Q, Xu LL, et al. Toll-like receptor 4 signaling inhibits malignant pleural effusion by altering Th1/ Th17 responses. Cell Biol Int 2015; 39: 1120-30.

32. Loser K, Vogl T, Voskort M, et al. The Toll-like receptor 4 ligands Mrp8 and Mrp14 are crucial in the development of autoreactive CD8+ T cells. Nat Med 2010; 16: 713-7.

33. $\mathrm{Mu} \mathrm{HH}$, Hasebe A, Van Schelt A, Cole BC. Novel interactions of a microbial superantigen with TLR2 and TLR4 differentially regulate IL-17 and Th17-associated cytokines. Cell Microbiol 2011; 13: 374-87.

34. Bhan U, Ballinger MN, Zeng X, Newstead MJ, Cornicelli MD, Standiford TJ. Cooperative interactions between TLR4 and TLR9 regulate interleukin 23 and 17 production in a murine model of gram negative bacterial pneumonia. PLoS One 2010; 5: e9896.

35. Banus S, Stenger RM, Gremmer ER, et al. The role of Tolllike receptor-4 in pertussis vaccine-induced immunity. BMC Immunol 2008; 9: 21. 